\title{
Primary schwannoma of the thyroid gland involving the isthmus: report of a case
}

\author{
Giuseppa Graceffa $\cdot$ Calogero Cipolla $\cdot$ \\ Ada Maria Florena • Irene Gentile • \\ Giancarlo Pompei · Mario A. Latteri
}

Received: 28 July 2011/Accepted: 3 November 2011/Published online: 24 May 2012

(c) Springer 2012

\begin{abstract}
Primary thyroid schwannomas are extremely rare tumors and there are very few reports of such tumors in the literature. This report presents a rare case of schwannoma involving the isthmus of the thyroid in a 47-year-old male, presenting as a symptomatic predominating cold nodule within a multinodular goiter. The patient underwent total thyroidectomy. The histological examination indicated an Antoni A-type schwannoma. The clinical, radiological and pathological findings of the tumor are discussed, emphasizing the difficulty in reaching a correct preoperative diagnosis. Only 18 cases of primary schwannoma of the thyroid gland have so far been described in the literature and, this is only the second report of thyroid schwannoma localized in the isthmus.
\end{abstract}

Keywords Schwannoma - Thyroid gland

\section{Introduction}

Schwannomas, also known as neurinomas or neurilemomas, are rare benign tumors of the peripheral and cranial nerves originating from the neuronal sheath cells (Schwann cells), which generally grow slowly. Although malignant degeneration has been reported, this is extremely rare [1].

G. Graceffa · C. Cipolla $(\bowtie) \cdot$ I. Gentile $\cdot$ M. A. Latteri Division of General and Oncological Surgery, Department of Oncology, A.O.U. Policlinico "Paolo Giaccone", Via del Vespro 129, 90127 Palermo, Italy

e-mail: calogerocipolla@virgilio.it

A. M. Florena - G. Pompei

Department of Human Pathology, A.O.U. Policlinico

"Paolo Giaccone", University of Palermo, Via del Vespro 129,

90127 Palermo, Italy
Schwannomas, first described in 1908 by Verocay, are classified into two histological types: Antoni A and Antoni B. The Antoni A regions are highly cellular with orderly dense cell arrangements. The nuclei present palisading, which results in nuclear-free zones composed of cytoplasmic processes arranged in fascicles known as Verocay bodies. Antony B areas are myxoid, less orderly, less cellular and may also contain microcysts.

Although schwannomas can occur anywhere in the body, from 25 to $45 \%$ originate in the head and neck region and most commonly involve the intracranial nerves or any of their branches. The eighth cranial nerve is the most frequent site involved, followed by the fifth and the seventh cranial nerves [2]. Primary schwannomas of other extracranial sites of the head and neck region are very rare. Such tumors may develop anywhere from the base of the skull to the thoracic inlet, but they are most commonly found in the middle area of the neck.

Schwannomas involving the thyroid gland are particularly rare. Eighteen cases have been reported in the literature, and the point of origin was the isthmus of the gland in only one case [3]. This report describes a rare case of schwannoma involving the isthmus of the thyroid gland, presenting as a symptomatic cold nodule.

\section{Case report}

A 47-year-old male was referred by an endocrinologist because of a thyroid nodule localized in the isthmus of the gland. The lesion was first observed about a year earlier, during the follow-up for a multinodular goiter (Fig. 1), and had showed a slow increase in size since then. The patient complained of recent dysphagia, odynophagia and hoarseness. A physical examination showed a non-tender, 
Fig. 1 US findings of multinodular goiter. a Left lobe; b isthmus; $\mathbf{c}, \mathbf{d}$ right lobe
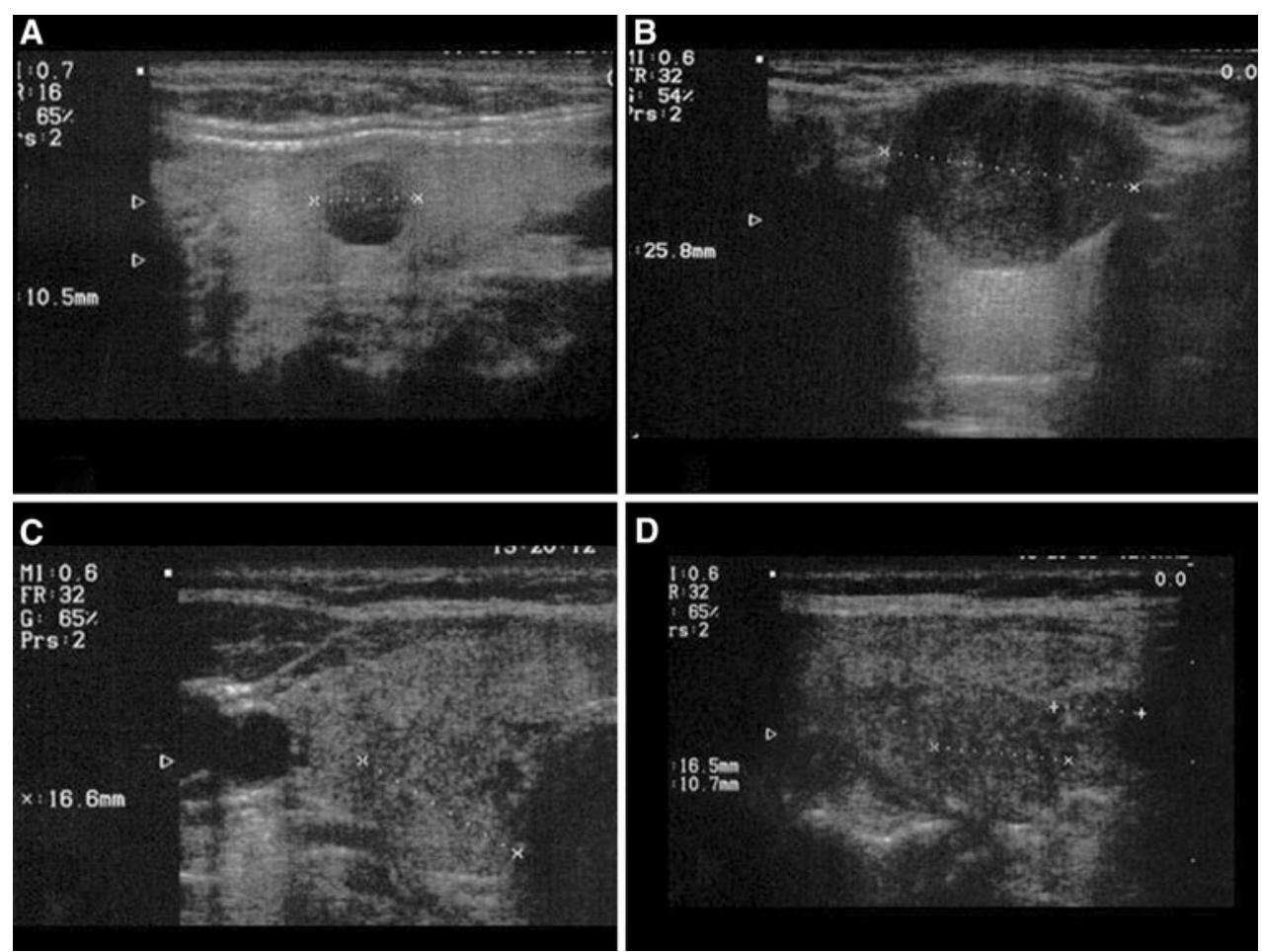

mobile nodule measuring approximately $2.5 \mathrm{~cm}$ in size with well-defined margins. There was no cervical lymphadenopathy. The laboratory results showed normal serum levels of fT3, fT4, and TSH and a concentration of thyroglobulin, calcitonin and antithyroid peroxidase antibody within normal limits. An ultrasonographic examination showed that the nodule was an oval, well-defined, hypoechogenic solid tumor localized in the isthmus of the thyroid, measuring $25.8 \mathrm{~mm}$ at its maximum diameter. The nodule was cold on a $\mathrm{Tc}_{99}$ thyroid scan (Fig. 2). Fineneedle aspiration findings of the isthmic nodule indicated a diagnosis of nodular goiter (Fig. 3). A flexible fiberoptic examination showed no laryngeal abnormalities and the vocal cords were bilaterally mobile.

The patient underwent total thyroidectomy with an uneventful postoperative course, and was discharged on the second postoperative day. Neither any transient nor definite injuries of the laryngeal recurrent nerves or of the parathyroid glands were observed.

\section{Pathology report}

A gross examination showed the right thyroid lobe to measure $4.0 \times 2.3 \times 1.8 \mathrm{~cm}$, and the left lobe $5.0 \times$ $3.0 \times 1.5 \mathrm{~cm}$, the isthmus was entirely occupied by a $2.5 \mathrm{~cm}$ nodule. Microscopic examination revealed an encapsulated nodule composed of spindle-shaped Schwann cells with little stromal matrix (Antony A type) and the presence of Verocay bodies. Immunohistochemical staining showed that the cells were strongly and diffusely positive for S-100, weakly positive for vimentin, while they were negative for CD34, actinin, desmin and CD99 (Fig. 4).

\section{Discussion}

Non-epithelial thyroid tumors are extremely rare, representing $<1 \%$ of all thyroid tumors [4]. They may include lymphomas, teratomas, lipomas, hemangiomas and schwannomas. A primary schwannoma of the thyroid was first reported in 1964 by Delaney and Fry [5], although the first description of the schwannoma as a non-epithelial tumor arising in the thyroid gland was made by Frantz in 1962 [6]. Their origin from the intrathyroidal sensory nerves or sympathetic and parasympathetic innervation has been postulated [7]. A review of the pertinent literature produced only 18 cases of primary schwannomas of the thyroid, mostly reported as single case reports. Almost all the reported cases involve the right lobe of the thyroid; a few cases are reported involving the left lobe, and only in one case does the tumor involve the isthmus of the gland [3].

It is extremely difficult to reach a correct preoperative diagnosis of thyroid schwannoma. The first sign noticed by a patient is a mass in the lower neck and only in a few 


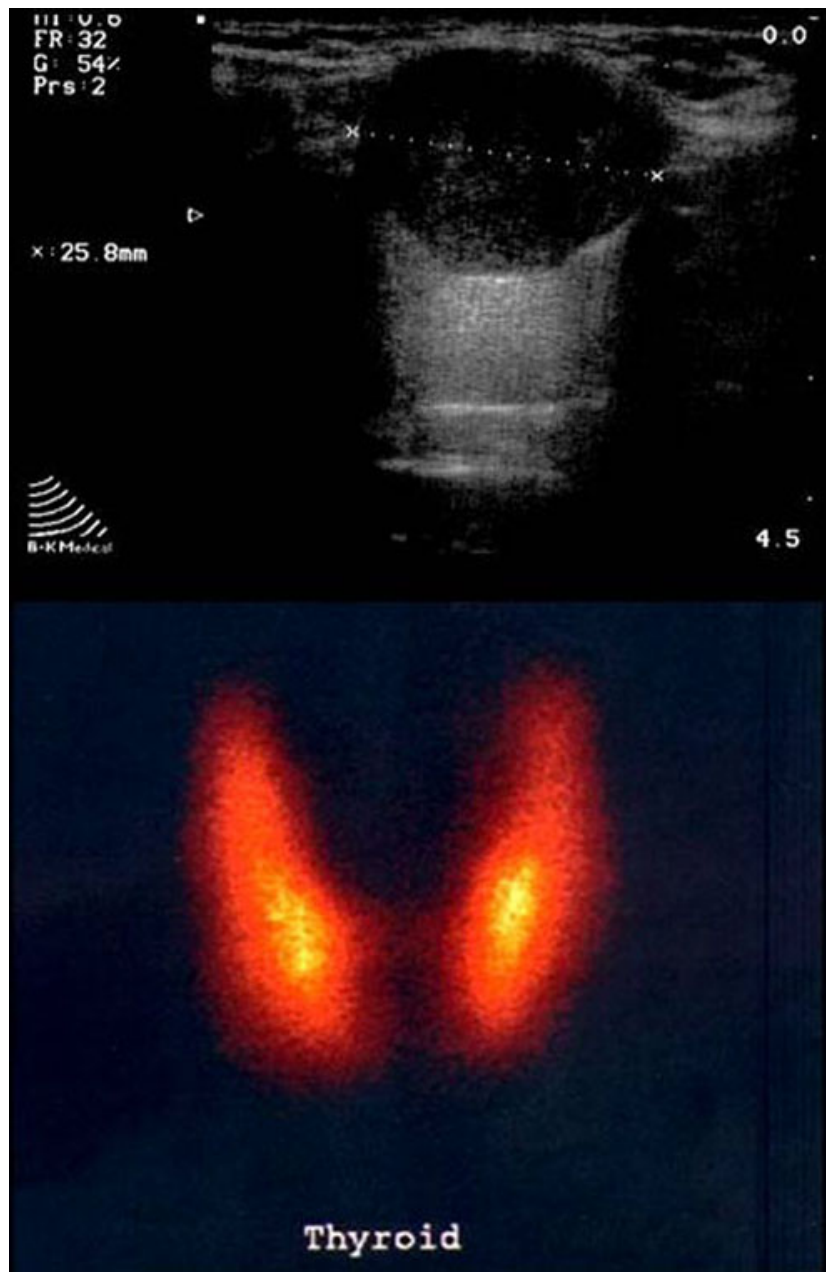

Fig. 2 Ultrasonographic and scintigraphic patterns of the thyroid nodule

reported cases do patients complain of local neck symptoms. One patient with a large tumor of the right lobe stretching as far as the retrosternal space presented respiratory distress [8]. One patient complained of a flushed face and fatigue as the main reason for medical consultation [9]. Another patient with a tumor originating from the isthmus suffered from hoarseness [3], similar to the current patient, who also complained of dysphagia and odynophagia.

The serum levels of thyroid hormones were within the normal range in all cases but one. One patient was treated with L-thyroxin because of a primary hypothyroidism due to Hashimoto's thyroiditis [10].

Ultrasonography shows thyroid schwannomas as a welldelineated, solid or predominantly solid tumor of diminished echogenicity, without any involvement of the cervical lymph nodes. Diagnostic support by thyroid scintigraphy is not necessary. Thyroid scintigraphy in seven of the cases showed schwannomas to appear as a cold area within the affected lobe, as was observed in the current case.

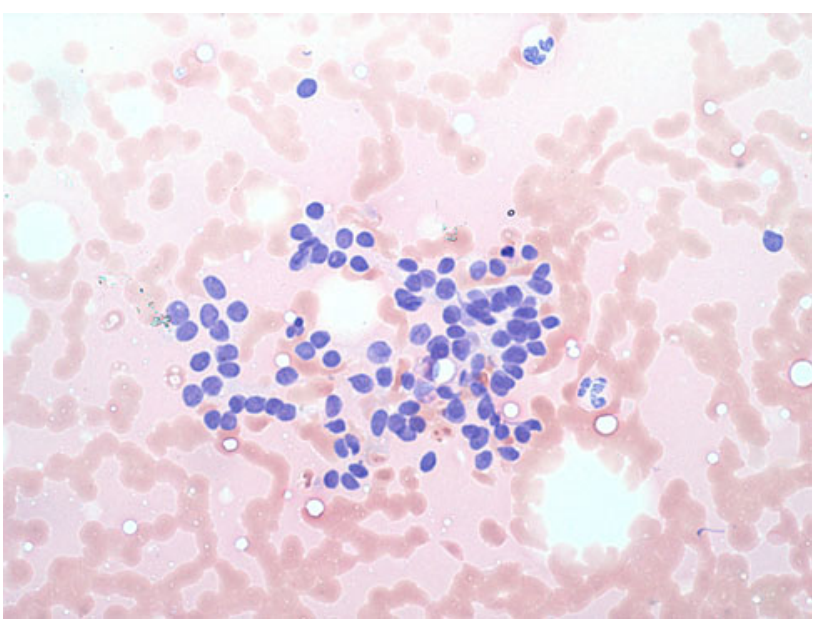

Fig. 3 Cluster of typical thyrocytes with nude nuclei in a focal follicular aggregation

Only a few of the reported patients underwent computed tomography $(\mathrm{CT})$ of the head and neck $[6,9,11]$, but this modality did not provide any specific features. The CT appearance of schwannoma is usually a well-circumscribed homogeneous mass of soft tissue density. Although the CT findings of inhomogeneity may suggest malignancy in neurogenic tumors [12], benign schwannomas have sometimes been reported to be non-homogenous on CT examination $[9,13]$.

The typical cytological features of schwannoma include the presence of cells with slender wavy nuclei, fibrillary stroma, nuclear palisading and the presence of Verocay bodies. S-100 and CD-34 positive immunostaining may aid in the diagnosis of neural sheath tumors and may be mandatory for distinguishing schwannomas from other tumors such as leiomyomas and solitary fibrous tumors. Fine needle aspiration biopsy was performed preoperatively in 6 of the reported cases and a precise diagnosis of schwannoma was made in only two patients $[14,15]$. The cytological examination performed in the current patient was consistent with the diagnosis of nodular goiter.

Surgical excision is considered to be curative for schwannoma. Most of the cases reported in the literature underwent lobectomy or enucleation, with an excellent prognosis. The current patient underwent total thyroidectomy because of the multinodular goiter and complications due to local neck symptoms.

\section{Conclusions}

Primary thyroid schwannomas are extremely rare, and their location in the isthmus of the thyroid is even less common. This report presented only the second reported case of a 
Fig. 4 a The neoplasia is surrounded by an evident capsular structure that clearly divides it from the surrounding thyroid parenchyma $(\times 250)$. b Verocay bodies typical of a schwannoma $(\times 400)$. c Mucous areas with myxoid-type cellular density. d Positive immunohistochemical staining with S-100 indicates the nervous nature of the neoplastic elements $(\times 400)$
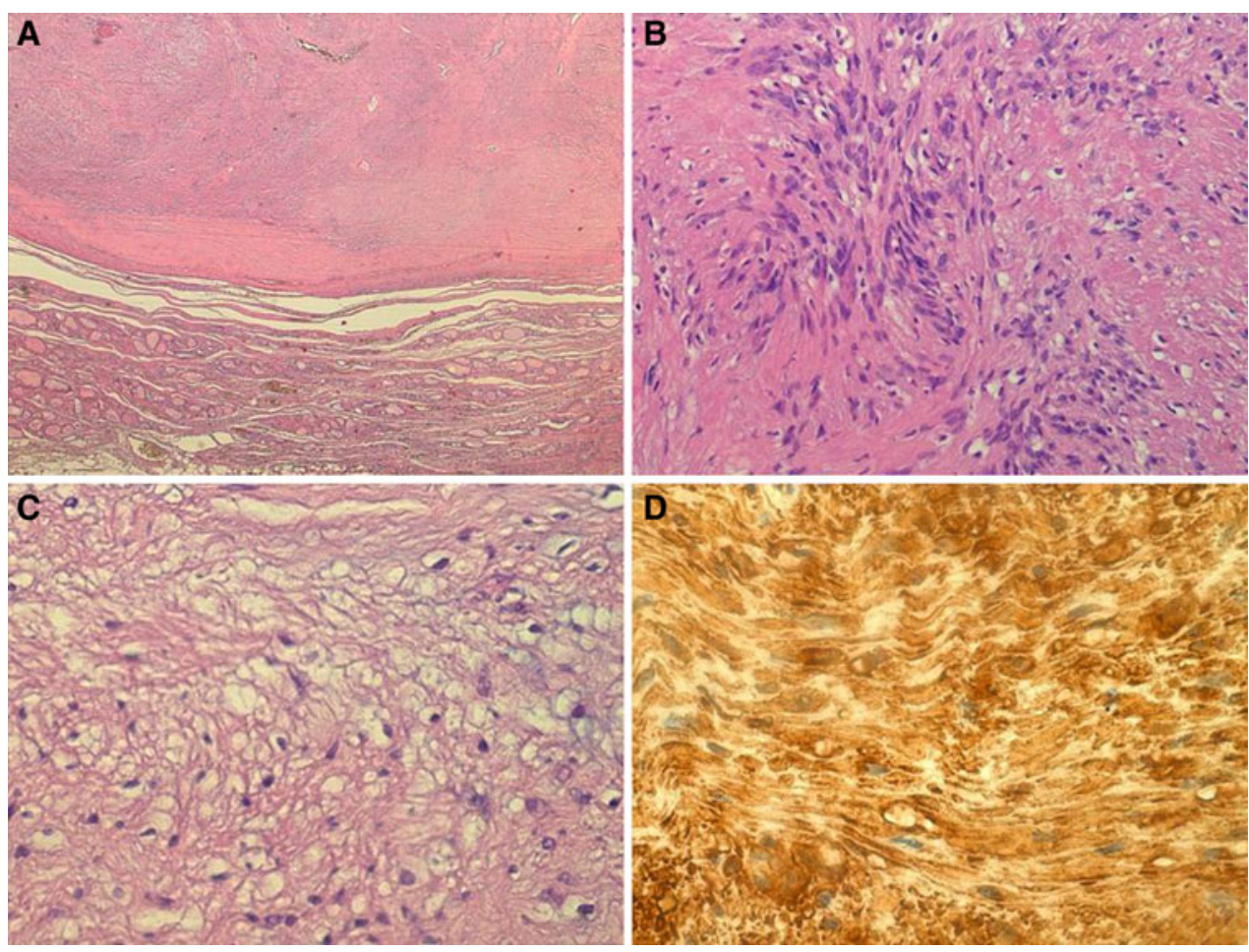

primary schwannoma involving the isthmus of the thyroid gland; the first case was reported by Iizuka et al. [3] in 1978. In spite of their rarity, schwannomas, like other nonepithelial thyroid tumors, should be taken into consideration in the preoperative differential diagnosis of thyroid nodules, particularly in the presence of a single thyroid nodule. Complete surgical excision is considered to be a curative therapeutic strategy and would appear to be the treatment of choice.

Conflict of interest Giuseppa Graceffa, Calogero Cipolla, Ada Maria Florena, Irene Gentile, Giancarlo Pompei and Mario A. Latteri declare that they have no conflict of interest.

\section{References}

1. Calcaterra TC, Wang MB, Sercarz JA. Unusual tumors. In: Myers EN, Suen JY, editors. Cancer of the head and neck. 3rd ed. Philadelphia: Saunders; 1996. p. 664-9.

2. Sagar SM, Israel MA. Primary and metastatic tumors of the nervous system. In: Braunwald E, Fauci AS, Kasper DL, editors. Harrison's principles of internal medicine. 15th ed. New York: McGraw-Hill; 2001. p. 2442-52.

3. Iisuka K, Togawa K, Konno A, Higasi K. A neurilemoma of the thyroid gland. Jibiinkouka. 1978;50:1077-80.

4. Million RR, Cassisi NJ. Management of head and neck cancer. 2nd ed. Philadelphia: Lippincott; 1994. p. 785-810.
5. Delaney WE, Fry KE. Neurilemmoma of the thyroid gland. Ann Surg. 1964;160:1014-6.

6. Frantz VK. Pathology of the thyroid. In: Warner SC, editor. The thyroid: a fundamental and clinical text. 2nd ed. New York: Harper and Row; 1962. p. 321.

7. Thompson LD, Wenig BM, Adair CF, Heffes CS. Peripheral nerve sheath tumors of the thyroid gland: a series of four cases and a review of the literature. Endocr Pathol. 1996;7:309-18.

8. Goldstein J, Tovi F, Sidi J. Primary schwannoma of the thyroid gland. Int Surg. 1982;67:433-4.

9. Sugita R, Nomura T, Yuda F. Primary schwannoma of the thyroid gland: CT findings. AJR Am J Roentgenol. 1998;171:528-9.

10. Uri O, Baron E, Lefel O, Bitterman A. Primary schwannoma of the thyroid gland presenting as an asymptomatic cold nodule. Am J Otolaryngol. 2009;30:427-9.

11. Andrion A, Bellis D, Delsedime L, Bussolati G, Mazzucco G. Leiomyoma and neurilemmoma. Report of two unusual nonepithelial tumours of the thyroid gland. Virchows Arch A Pathol Anat Histopatol. 1988;413:367-72.

12. Coleman BG, Arger PH, Dalinka MK, Obringer AC, Raney BR, Meadows AT. CT of sarcormatous degeneration in neurofibromatosis. AJR Am J Roentgenol. 1983;140:383-7.

13. Hamza A, Fagan JJ, Weissman JL, Myers EN. Neurilemomas of the parapharyngeal space. Arch Otolaryngol Head Neck Surg. 1997; 123:622-6.

14. Baglaj M, Markowska-Woyciechowska A, Sawicz-Birkowska K, Dorobisz U. Primary neurilemmoma of the thyroid gland in a 12-year-old girl. J Ped Surg. 2004;39:1418-20.

15. Jayaram G. Neurilemmoma (schwannoma) of the thyroid diagnosed by fine needle aspiration cytology. Acta Cytol. 1999;43: 743-4. 